\title{
Acción colectiva territorial en el contexto fronterizo México-Guatemala. Productores de limón persa del Distrito de Riego San Gregorio
}

\section{Territorial Collective Action in a Mexico-Guatemala Border Context. Persian Lemon Producers of the San Gregorio Irrigation District}

\author{
Francisco F. Herrera López* (iD) https://orcid.org/0000-0002-2749-9011 \\ Héctor B. Fletes Ocón ** (iD https://orcid.org/0000-0001-5603-1808 \\ Guillermo S. Valdiviezo Ocampo *** (D) https://orcid.org/0000-0001-5007-0398
}

\section{Resumen}

El objetivo de este artículo es examinar los mecanismos de acción colectiva que implementaron los pequeños productores de limón persa en el Distrito de Riego San Gregorio en la región fronteriza México-Guatemala. El análisis de las estrategias que estos actores desarrollaron en las áreas de producción, difusión de conocimientos y comercialización, revela las formas en que enfrentan los retos del desarrollo territorial y los cambios en el sector alimentario. Debido a que la producción de este cítrico es un proceso reciente en el territorio, no se cuentan con estadísticas específicas y estudios previos para tomarlos como referencia, por lo que se parte de un estudio exploratorio para realizar una metodología de perspectiva cualitativa basada en la observación participante, entrevistas a distintos actores locales, conocimiento de la historia oral, aplicación de una encuesta a pequeños productores y la consulta de bases de datos. Se encontró que, para contender frente a la reestructuración del sector alimentario, los productores locales recuperaron elementos endógenos del territorio, lo que les permitió cooperar y coordinarse mediante organizaciones y la acción colectiva. Con ello adoptaron y adaptaron a sus condiciones un programa externo de introducción de un nuevo cultivo. Al ser un proceso reciente, se cuentan con pocos estudios de limón en la región.

Palabras clave: territorios rurales; globalización; pequeños productores; comercialización; frontera México-Guatemala.

Cómo citar: Herrera López, F. F., Fletes Ocón, H. B., y Valdiviezo Ocampo, G. S. (2020). Acción colectiva territorial en el contexto fronterizo México-Guatemala. Productores de limón persa del Distrito de Riego San Gregorio. región y sociedad, 32, el391. doi: I0.22I98/ rys2020/32/1391

*Autor para correspondencia. Investigador independiente, especialista en temas de desarrollo local. Correo electrónico: pako_299@hotmail.com

\section{Abstract}

The aim of this paper is to examine the collective action mechanisms implemented by the small Persian lemon producers in the San Gregorio Irrigation District in the Mexico-Guatemala border region. The analysis of the strategies that these actors developed in the production, dissemination of knowledge, and commercialization areas reveals the way in which small producers face the challenges of territorial development and changes in the food sector. Because the production of this citrus fruit is a recent process in the territory, there are no specific statistics and previous studies to take them as a reference, so it starts with an exploratory study to later carry out a qualitative perspective methodology based on participant observation interviews with different local actors, knowledge of the oral history, the application of a survey to the small producers, and the consultation of databases. It is found that to fight against the restructuring in the food sector, the local producers recovered endogenous elements to the territory, which allowed them to cooperate and coordinate through organizations and collective action. With this, they adopted and adapted to their conditions an external program to introduce a new crop.

Keywords: rural territories; globalization; smallholders; commercialization; Mexico-Guatemala border.

\footnotetext{
** Universidad Autónoma de Chiapas, Facultad de Ciencias Sociales. Calle Presidente Obregón s. n., Col. Revolución Mexicana, C. P. 29200, San Cristóbal de Las Casas, Chiapas, México. Correo electrónico: hector.fletes@unach.mx

*** Universidad Autónoma de Chiapas, Facultad de Ciencias Sociales. Calle Presidente Obregón s. n., Col. Revolución Mexicana, C.P. 29200, San Cristóbal de Las Casas, Chiapas, México. Correo electrónico: gsvo67@gmail.
}

Recibido: 29 de agosto de 2020

Aceptado: 26 de noviembre de 2020

Liberado: 30 de diciembre de 2020 


\section{Introducción}

Robinson (2015, p. 75) y Sekine y Bonanno (2016, pp. 18 y 52-59) han documentado que desde finales de la década de 1970 el sector agroalimentario del mundo entero se encuentra inmerso en procesos de globalización, a través de los cuales los sistemas de producción agrícola nacionales y la alimentación se vinculan, de uno u otro modo, con una estructura organizada a escala global. La división internacional del trabajo agrícola, el alargamiento de las cadenas de mercancías y el control oligopólico que ejercen grandes empresas transnacionales en diferentes nodos de las cadenas productivas, han reducido la proporción de los ingresos que los productores se apropian y con ello comprometen la capacidad de la actividad agrícola de cumplir la función de asegurar la producción de alimentos y reducir la pobreza (Flores, 2017, pp. 29-30; Maya, 2017, p. 16; Torres, 2017, p. 126).

En México, las políticas de desregulación, privatización y liberalización comercial han afectado en gran medida a pequeños y medianos productores que han reducido o abandonado la producción de cereales, sobre todo de maíz, o han optado por otras actividades o por la migración (Eakin, Sweeney, Lerner, Appendini, Perales, Steigerwald, Dewes, Davenport y Bausch, 2018, p. 46; Trápaga, 2017, p. 74). Lo anterior conduce a la reflexión sobre los retos que enfrentan los territorios con vocación agrícola y las acciones de su población frente a un entorno de cambio económico y político.

En Chiapas, a partir de la administración estatal del periodo 2006-2012, se estableció la política de reconversión productiva, cuyo eje de orientación para la agricultura estatal fue la promoción de cultivos de alto valor agregado. La intención fue sustituir la producción de maíz (Fletes, Ocampo y Valdiviezo, 2018). En distintas regiones del estado, este programa conllevó la siembra de tomate, piñón, palma africana y limón persa. En el Distrito de Riego San Gregorio de Chiapas (DRSG), ${ }^{1}$ ubicado entre los municipios de Frontera Comalapa y La Trinitaria, el clima tropical y la disponibilidad de agua son las condiciones que permitieron la promoción oficial del limón persa, desde mediados de la primera década de 2000, entre los pequeños productores (PP). De este modo, esos actores se encontraban con los retos de superar las caídas de precios en los cultivos básicos e implementar nuevos y desconocidos procesos técnicos asociados con el limón persa. La presente investigación tiene la finalidad de examinar las estrategias de acción colectiva que implementaron dichos productores en las áreas de la producción, la difusión de conocimientos y la comercialización en torno al nuevo producto que la reconversión productiva estableció.

El trabajo contribuye al conocimiento de las acciones colectivas que están realizando los PP para contender ante el estancamiento relativo que tiene la actividad agrícola en comparación con el resto de la economía. El análisis se

1 El DRSG conforma, junto con los distritos de riego de Cacahoatán-Suchiate, Río Blanco y Cuxtepeques (todos de Chiapas), el Organismo de Cuenca XI Frontera Sur, mediante el cual la Comisión Nacional del Agua (CONAGUA) gestiona y administra la política hidráulica en el país. También se conoce como San Gregorio Chamic, por la localidad contigua a la carretera federal, donde se ingresa a un grupo numeroso de localidades que conforman el Distrito. 
realiza en un territorio de la frontera sur de México que ha sido poco analizado. En el contexto de la política de reconversión, se conecta el proceso de globalización económica con la emergencia de un sistema productivo regional que a su vez evidencia relaciones económicas y sociales entre México y Centroamérica. Sobre todo, se documentan las relaciones productivas y comerciales de un producto frutícola que también ha sido poco estudiado en México. Por último, es relevante el conocimiento sobre el potencial endógeno y la capacidad de agencia que existe en espacios agrarios a partir de la colaboración entre los actores y mediante las acciones colectivas frente a la profundización de la competencia y de la globalización.

La estructura del documento consiste en lo siguiente. Primero, se presentan la metodología de enfoque cualitativa utilizada para la realización de la investigación basada en observación participante, entrevistas semiestructuradas a diversos actores locales, conocimiento de la historia oral y encuestas. Después se discuten los cambios relacionados con la globalización del sector agroalimentario, así como el cambio político neoliberal que reformuló las condiciones para la agricultura en México y para los PP. A raíz de este conjunto de procesos de diferente escala, se han producido retos mayúsculos para los territorios rurales y los PP, por lo que se subraya el papel de la acción colectiva y de las organizaciones para enfrentarlos. Enseguida, se presenta un breve panorama del contexto productivo en el que se desarrolla este estudio de caso. Después, se analiza la introducción y la expansión del limón persa en el Distrito a través de la interrelación de diversos actores. A continuación, se examinan las prácticas de producción implementadas y el papel central que los PP asumieron en su difusión e innovación, lo que resultó en la agricultura intensiva con impacto en el ambiente. En el penúltimo apartado se examina la organización y las redes de relaciones que los productores establecieron para coordinar la compra y la venta del producto con intermediarios foráneos. Al final, se presentan las conclusiones.

\section{Metodología}

En cuanto que análisis de un sistema productivo y sus actores, la perspectiva teórica se basó en el concepto de campo agroalimentario (González, 2020) como escenario amplio de las estrategias de acción colectiva. Esta noción plantea que los actores compiten por los beneficios producidos en diferentes nodos de las cadenas alimentarias. Se trata de "un sistema abierto de relaciones sociales, donde participan una variedad de actores sociales, con motivos particulares, diferentes formas de poder y escalas de valor, por lo que ejercen asociaciones e influencias mutuas" (p. 166).

La investigación tiene una perspectiva cualitativa y se complementa con la aplicación de una encuesta, método que han validado otros estudios (González de la Rocha y Villagómez, 2008). El trabajo de campo se desarrolló en 12 localidades del DRSG entre junio de 2015 y julio de 2016, con posteriores seguimientos de campo y documentales en 2017 y 2020. Se entrevistó a funcionarios 
de la Secretaría del Campo de Gobierno del Estado de Chiapas (Delegación III Fronteriza). Se realizaron varias entrevistas semiestructuradas a tres integrantes de la mesa directiva del Comité Regional del Sistema Producto Limón Persa del Distrito de Riego de San Gregorio (CRSPL), ${ }^{2}$ quienes son PP con residencia en la localidad de Nuevo Villaflores y realizan actividades de comercialización de limón persa hacia Guatemala. Así, las entrevistas se enfocaron en la reconstrucción del proceso de difusión de dicha fruta y en el conocimiento de la dinámica de la comercialización.

Además, se entrevistó a dos comerciantes foráneos ubicados en el Distrito de Riego, al propietario de la Empacadora Benavides, ubicada en la localidad de San Gregorio, y al dueño de la Empacadora GONARCHI y de Citricultores de Valle de Chamic, S. C. de R. L. de C. V., ubicada en Flor de Mayo, ambas empresas vinculadas a la comercialización del limón persa hacia Veracruz y Tabasco, respectivamente. Se entrevistó también al personal técnico de la Campaña contra Huanglongbing (HLB) de los Cítricos, del Comité Estatal de Sanidad Vegetal (CESAVE) de Chiapas. ${ }^{3}$

Así mismo, se realizó observación participante en el proceso de venta del limón persa con intermediarios de Veracruz. Se asistió a un taller que organizó la Junta Local de Sanidad Vegetal III Fronteriza enfocado en el control de la mosca prieta y en el uso de plaguicidas. Al terminar la actividad, se registró la percepción de algunos asistentes sobre el tema. Por otra parte, se asistió a dos asambleas generales de la Cooperativa de Citricultores San Isidro, en la localidad de Nuevo Villaflores. Distintos diálogos informales permitieron conocer una historia oral acerca del conjunto de relaciones sociales y comerciales en torno al limón persa.

La encuesta se realizó a 70 productores de limón persa de 12 localidades del Distrito. El objetivo fue identificar sus condiciones y relaciones productivas y comerciales. La determinación del tamaño de la muestra se basó en la aplicación de la fórmula:

$$
n=\frac{\left(N * Z^{2 *} P^{*} Q\right)}{\left(s^{2} *(N-1)+Z^{2 *} P^{*} Q\right.}
$$

En donde la población $N$ es igual a 300 PP; el nivel de confianza es de $95 \%$ y corresponde a un valor de $Z$ de 1.96 . El margen de error (s) es de $6 \%$. Dado que en las entrevistas y en el muestreo piloto se observó que se trataba de una población homogénea en términos relativos, se consideró un valor de la proporción esperada o proporción de éxito $(P)$ de $90 \%$ y una probabilidad de fracaso $Q$

2 El CRSPL es una organización perteneciente al Comité Estatal del Sistema Producto Limón Persa, integrada y presidida por productores de limón persa de este territorio, que tiene el objetivo de gestionar recursos y promover la organización territorial para la realización de acciones en conjunto.

3 EI CESAVE es un organismo auxiliar de la Secretaría de Agricultura, Ganadería, Desarrollo Rural Pesca y Alimentación (SADER) y del Servicio Nacional de Sanidad, Inocuidad y Calidad Agroalimentaria (SENASICA) que se encarga de la detección, el control y la erradicación de plagas y enfermedades de los cultivos, con el objetivo de fomentar la fitosanidad e inocuidad para que los productores puedan comercializar sus productos a escala nacional e internacional. 
igual a $1-P$, de $10 \%$. Para la selección de los elementos, se realizó un muestreo estratificado aleatorio, calculado de manera proporcional por localidad. Cabe mencionar que la encuesta se utiliza aquí para tener referencias básicas de los productores, ya que la prioridad es reconstruir, a través de las entrevistas, de la observación participante y de la historia oral el campo agroalimentario en cuestión. Las dimensiones de análisis de la acción colectiva se enfocan en tres aspectos principales: la organización para la producción, la difusión de conocimientos y las estrategias en el ámbito de la comercialización.

\section{Globalización, cambios en la política agroalimentaria y el papel de la acción colectiva}

En el sector agroalimentario, la globalización se ha manifestado en la integración de los procesos de producción, transformación y comercio, en la cual las corporaciones y los estándares globales repercuten en las prácticas agrícolas y en el desenvolvimiento de los territorios (Bonanno y Wolf, 2018, p. 3; Garrapa, 2018, pp. 158-159; Guzmán, 2018). En este proceso histórico, se ha profundizado una división internacional del trabajo agrícola en la cual los países desarrollados, como Estados Unidos, la mayoría de los países europeos y Nueva Zelanda, se constituyeron en exportadores de granos básicos, cárnicos y lácteos a los países en vías de desarrollo, con lo cual los últimos perdieron su soberanía alimentaria al importar hasta 65 millones de toneladas de cereales a finales de la década de 1960 y al especializarse en la exportación de frutas y hortalizas (FH) (Biel, 2007 y 2016; Maya, 2017; Robinson, 2015). Además, se han establecido relaciones asimétricas entre el sector productor y otros nodos de las cadenas agroalimentarias (Mutonyi, 2019; Ochieng, Knerr, Owuor y Ouma, 2018; Reig, 2004).

Sin embargo, hay evidencia de que los impactos de los procesos globales se filtran debido a las condiciones institucionales, socioculturales y ambientales a diferentes escalas, e incluso hay la certeza de que los procesos locales pueden mediar o amplificar su influencia (Eakin et al., 2018, p. 44). En México, a partir de la liberalización comercial implementada desde la década de 1980 y al firmarse el Tratado de Libre Comercio de América del Norte (TLCAN), se impulsó la especialización productiva en $\mathrm{FH}$, productos con "ventajas comparativas", mientras que los cereales cedieron terreno (Luiselli, 2017, pp. 237-238). Esta situación propició un cambio en la estructura de la producción: "el valor se modificó en favor de los productos para la exportación más rentables" (Puyana y Romero, 2008, p. 49). Resultados contradictorios de estas reformas, como el aumento de la ocupación en el sector y una recuperación positiva de la balanza comercial agropecuaria, se explican por el alza internacional de precios, los subsidios que recibieron en algunos grupos de productores, la depreciación del peso frente al dólar estadounidense y el uso de mano de obra accesible en parte por la reducción de la emigración rural (Yúnez, 2018, pp. 104-111).

Con todo, este proceso perjudicó en gran medida a los pequeños y medianos productores, porque el cambio en los programas de fomento y en los encadena- 
mientos que durante décadas les había permitido obtener cierto nivel de rentabilidad y excedentes para contribuir al abastecimiento del mercado nacional, les significó condiciones muy difíciles para competir con los alimentos importados (Fitting, 2014). Entre los retos que tienen los PP que han apostado por la producción de $\mathrm{FH}$, se encuentran los altos costos de inversión y de tecnología, así como el control que las corporaciones globales tienen del sistema de distribución, de las centrales de abasto y de las tiendas de autoservicio (Castillo, Ayala, Jiménez y Vargas, 2014; Macías, 2017).

Es importante señalar que los imperativos económicos señalados tienen configuraciones, efectos y respuestas diferenciadas en las distintas regiones (Bueno, 2016). En este sentido, se puede identificar otro tipo de retos que enfrentan los PP y los territorios en que se desenvuelven, más de carácter socioespacial y no sólo sectorial. En su trabajo sobre el desarrollo endógeno en territorios que denomina "frágiles" (aunque no lo explica, se refiere a territorios rurales del sur global con actividades agrícolas), Vázquez (2015) enfatiza un cúmulo de retos que enfrentan estos espacios, como "una fuerte presión sobre el uso del suelo" y "la introducción de nuevos métodos de producción y formas de gestión de la actividad productiva" (p. 187).

En las décadas de 1980 y 1990, las políticas de desarrollo en América Latina $(\mathrm{AL})$ se instrumentaban de una manera sectorizada y vertical, lo que llevaba a la necesidad imperante de la "organización de los distintos niveles territoriales y la movilización tendiente a consolidar la democracia” (Arocena y Marsiglia, 2017, pp. 38 y 291). De acuerdo con Vázquez (2015), los trópicos de AL son un caso de particular interés, pues se trata de territorios donde las instituciones extractivas han producido excedentes elevados que no se reinvirtieron, sino que se repartieron entre las élites económicas y políticas. Además, los territorios con baja densidad de población, donde las actividades agrarias que utilizan recursos naturales son dominantes, han sido sometidos a una cierta discriminación en las políticas económicas, pues la prioridad ha sido el desarrollo urbano. Las áreas rurales acumulan graves problemas estructurales, ya que las explotaciones y empresas tienden a operar con bajos niveles de productividad, sus productos compiten con dificultad en los mercados, la baja conectividad espacial dificulta el acceso a los mercados, carecen de las infraestructuras suficientes y sus servicios sociales son limitados (Vázquez, 2015, pp. 199, 201-202).

En este contexto de cambio económico y político, una opción para las poblaciones rurales y agrícolas es la activación de la capacidad de agencia, la articulación y la cooperación orientada a la consolidación de instituciones, la transferencia de conocimiento local, la participación social y económica y la mejora de sus condiciones de vida (Arocena y Marsiglia, 2017). Una noción que ha destacado en este acercamiento al desarrollo territorial es la acción colectiva, entendida como "una construcción social generada por actores relativamente autónomos, para el logro de objetivos comunes con orientaciones diferentes, pero potencialmente dispuestos a cooperar" (Lugo-Morin, 2013, p. 159). Se propone también como una acción tomada por un grupo (ya sea de manera directa o en su nombre por una organización) en la búsqueda de los intereses compartidos de los miembros (Ochieng et al., 2018, p. 4). 
Estas perspectivas resaltan la cualidad de agencia de los individuos, así como una racionalidad económica en su actuación. Sin embargo, es importante entender que hay condiciones que son dadas a los actores. Melucci (1991) considera la acción colectiva como el "resultado de intenciones, recursos y límites, con una orientación construida por medio de relaciones sociales dentro de un sistema de oportunidades y restricciones" (pp. 357- 358). Las acciones colectivas se refieren no sólo al mercado, o a una racionalidad económica estricta a la manera de Olson, sino que hay diferentes tipos de individuos que pueden tener preferencias de actuación no egoístas (Camacho, 2008, p. 39), es decir, que "tienen en cuenta los intereses de otras personas, así como los propios, al tomar decisiones" (Lugo-Morin, 2013, p. 159). De ahí que la acción colectiva no responde sólo a una solución de mercado; también surge de la comunidad, es decir, de valores y conocimientos comunes (Francisco, 2010, p. 2).

En la literatura sobre los PP se señala una estrecha vinculación entre la capacidad colectiva y las organizaciones. De hecho, la acción colectiva "se institucionaliza en la forma de organizaciones de agricultores" (Ochieng et al., 2018 , p. 2). Según Mutonyi (2019, p. 2), la acción colectiva a través de las organizaciones de productores puede ayudar a los PP a tener acceso a los mercados domésticos y a los internacionales, a través de economías de escala, y adquirir un poder creciente para negociar y obtener mejores precios. Los grupos de productores pueden acortar las cadenas de comercialización al conectarse de manera directa con los mercados, eliminar intermediarios, reunir recursos y mejorar la coordinación de las actividades en las cadenas alimentarias (Mutonyi, 2019, p. 3; Ochieng et al., 2018, p. 2). ${ }^{4}$

\section{Contexto regional y productivo del DRSG}

Es importante entender el proceso de cambio productivo reciente que antecede a los intentos de establecimiento del limón persa y otras frutas en el DRSG. Dicho Distrito está ubicado entre los municipios de La Trinitaria y Frontera Comalapa, a tan sólo 20 kilómetros de la frontera con Guatemala, e integra una región fronteriza que tiene como centro socioeconómico y político regional la ciudad de Comitán de Domínguez. Se observa así una intensidad de relaciones comerciales entre la región y Guatemala, así como la participación de trabajadores centroamericanos en actividades agrícolas en ciertos cultivos, como el tomate verde.

En el DRSG en el año agrícola 2015-2016 operan 2511 usuarios, de los cuales 2369 son ejidatarios (con una superficie sembrada promedio de 5.5 ha, de las cuales se riegan 2.1 ha en promedio), y 142 propietarios privados (con una

4 Este texto comparte con el de Tisenkopfs, Adamsone-Fiskovica, Kilis, Šūmane, Grivins, Pinto-Correia y Bjørkhaug (2020) una perspectiva territorial en el estudio de los PP. Estos autores recurren al concepto de territorial fitting, entendido como "las estrategias y acciones que las granjas individuales llevan a cabo con el objetivo general de asegurar la sustentabilidad [...] de la granja, haciendo uso activo de los activos y conexiones territoriales disponibles, y agrupándolos en varias escalas" (p. 2). No obstante, en el presente trabajo se atiende la configuración colectiva, no individual, de estas iniciativas. 
superficie sembrada de 9.8 ha, de las que se riegan 6.3 ha en promedio) (Secretaría del Medio Ambiente y Recursos Naturales [SEMARNAT] y Comisión Nacional del Agua [CONAGUA], 2017, pp. 253 y 370). Algunas de las características de producción se encuentran en la tabla 1. Se observa que hay predominio de siembra de maíz en grano, con 13050 ha en el año agrícola 2015-2016, mientras que de limón se reportaron 230 ha. También es destacable que el DRSG muestra elevados rendimientos de los cultivos en comparación con el resto de la región. De maíz en grano se obtuvieron 6.5 ton/ha, mientras que de limón, 17 ton/ha en el año agrícola 2015-2016. En ese mismo año, en la región se obtuvieron 2.20 y 6.05 ton/ha, respectivamente.

Tabla 1. DRSG. Superficie sembrada y rendimiento por ha. Años agrícolas 2015-2016 y 2016-2017

\begin{tabular}{|c|c|c|c|c|c|c|}
\hline \multirow{2}{*}{ Ciclo } & \multirow[t]{2}{*}{ Modalidad } & \multirow[t]{2}{*}{ Cultivo } & \multicolumn{2}{|c|}{ Año agrícola 2015-2016 } & \multicolumn{2}{|c|}{ Año agrícola 2016-2017 } \\
\hline & & & $\begin{array}{l}\text { Superficie } \\
\text { sembrada }\end{array}$ & $\begin{array}{c}\text { Rendimiento } \\
\text { (ton/ha) }\end{array}$ & $\begin{array}{l}\text { Superficie } \\
\text { sembrada }\end{array}$ & $\begin{array}{c}\text { Rendimiento } \\
\text { (ton/ha) }\end{array}$ \\
\hline \multicolumn{3}{|c|}{ Total general } & 14500 & & 13640 & \\
\hline \multicolumn{3}{|c|}{ Otoño-invierno } & 2780 & & 2805 & \\
\hline & \multirow[t]{4}{*}{ Riego } & & 2780 & & 2805 & \\
\hline & & Maíz en grano & 2660 & 6.5 & 2695 & 7 \\
\hline & & Melón & 10 & 25 & nd & \\
\hline & & Otras hortalizas & 110 & 25 & 110 & 28 \\
\hline \multicolumn{3}{|c|}{ Primavera-verano } & 5960 & & 5940 & \\
\hline & \multirow[t]{2}{*}{ Riego } & & 1950 & & 1950 & \\
\hline & & Maíz en grano & 1950 & 6.5 & 1950 & 7 \\
\hline & \multirow[t]{2}{*}{ Temporal } & & 4010 & & 3990 & \\
\hline & & Maíz en grano & 4010 & 6.5 & 3990 & 7 \\
\hline \multicolumn{3}{|l|}{ Perennes } & 1330 & & 1330 & \\
\hline & \multirow[t]{4}{*}{ Riego } & & 1330 & & 1330 & \\
\hline & & $\begin{array}{c}\text { Estrella (zacate) } \\
\text { verde }\end{array}$ & 1080 & 55 & 1080 & 55 \\
\hline & & Limón & 230 & 17 & 230 & 18 \\
\hline & & Papayo & 20 & 55 & 20 & 55 \\
\hline \multicolumn{3}{|c|}{ Segundos cultivos } & 4430 & & 3565 & \\
\hline & \multirow[t]{2}{*}{ Temporal } & & 4430 & & 3565 & \\
\hline & & Maíz en grano & 4430 & 6.5 & 3565 & 7 \\
\hline
\end{tabular}

Fuente: SEMARNAT y CONAGUA (2017 y 2018). 
Sin embargo, como reflejo del proceso de reestructuración agroalimentaria que se ha impulsado a escala global y nacional, en los últimos veinte años en el DRSG se observan cambios en el patrón productivo, evidente en la reducción de la producción de maíz, al mismo tiempo que se expande la producción de diversas FH. Mientras que en 1990 se sembraron 18 mil hectáreas de maíz con una producción de 72 mil toneladas, en 2006 la superficie se había reducido a 1200 hectáreas, con una producción de 4500 toneladas (Rubio, 2006). Los cambios en la política agroalimentaria de México, a través de la apertura comercial, del retiro de los precios de garantía y de las empresas paraestatales de comercio, ocasionaron la caída de los precios y explican esta transformación drástica en la dinámica productiva.

El clima y las condiciones favorables para el cultivo de $\mathrm{FH}$, sumados a la crisis de la producción maicera, propició en los PP la búsqueda de alternativas productivas. Desde principios de la década de 1990, comenzaron a sembrar melón, lo cual llevó a que, en 1992, una empresa empacadora foránea se instalara en la región y se comprometiera a comprarles la fruta a altos precios mediante un sistema de crédito. No obstante, los productores fueron engañados: la empresa se llevó grandes cantidades de melón sin pagarles el producto. Después experimentaron un problema similar con la sandía: una empresa no les compró la fruta que había solicitado. Esta situación provocó la pérdida de confianza de los productores en la fruticultura y los obligó a intentar nuevas alternativas para enfrentar la caída de la rentabilidad del maíz, siempre y cuando recibieran apoyos gubernamentales y no invirtieran sus propios recursos ( $M$. López, comunicación personal, 11 de julio de 2015).

\section{Introducción y expansión del limón persa en el DRSG}

Se debe resaltar que la introducción y la difusión del limón persa implicaron la intervención de actores diversos, pero fueron los productores locales quienes establecieron la acción colectiva desde el territorio mediante la difusión de diversas técnicas de producción, la innovación sociotécnica, la cooperación, la coordinación y la participación activa en las redes comerciales.

De acuerdo con la información que proporcionaron directivos del CRSPL y varias asociaciones de citricultores de la zona, en 2016 se tenía un registro de 450 productores de limón persa en el Distrito, que en su conjunto sembraron una superficie aproximada de 1000 ha. Comparando este dato con la información de SEMARNAT y CONAGUA (2017), puede decirse que alrededor de 18\% de los 2511 productores del Distrito que utilizaron riego había adoptado el cítrico, cuya siembra abarcaba la sexta parte de las 6000 ha irrigadas.

Los citricultores se concentran en doce localidades: Nuevo Villaflores, Juan Sabines, Veinte de abril, El Paraíso, Flor de Mayo y Nuevo Llano Grande (municipio La Trinitaria), Revolución, San Gregorio, San Caralampio, Horizonte, Rodulfo Figueroa y Tamaulipas (municipio Frontera Comalapa). Según la encuesta aplicada, la mayor parte de los citricultores (94\%) cultiva menos de cinco hectáreas; mientras que $44 \%$ cultiva menos de dos. En $97 \%$ de los casos siembran 
también maíz. De las localidades mencionadas, destaca Nuevo Villaflores, que comenzó la siembra del limón persa en 2005. Ahí se ubican los comercializadores locales y la principal asociación de limoneros: la Cooperativa de Citricultores San Isidro, que posee la única empacadora de la región (véase figura 1).

Un docente de nivel básico que sembró tres hectáreas en Nuevo Villaflores introdujo el limón persa a principios de la década de 1990. Aunque esta persona motivó a que varios residentes del Distrito de Riego se dedicaran a producirlo, sólo un par de productores del Ejido Revolución decidieron invertir en su producción (Notas de campo). En 2002, representantes regionales de la Organización Proletaria Emiliano Zapata (OPEZ) promovieron el cultivo de limón, naranja y mandarina en diversas localidades e impartieron cursos de capacitación para que los productores instalaran un invernadero y aprendieran a realizar el injerto del limón persa. El impulso más claro de este cítrico comenzó en 2003, a partir de la visita que hizo un grupo de ingenieros del Instituto Interamericano para el Desarrollo Regional, A. C. a los PP de los ejidos Nuevo Villaflores y Flor de Mayo, para darles a conocer la aptitud de la región para la producción del limón persa y los mayores ingresos que traería consigo esta forma de diversificación productiva.

La falta de recursos económicos para adquirir los árboles de limón, combinada con las malas experiencias en la producción de frutas y la incertidumbre de los productores de la región en cuanto a invertir en un cultivo cuyo manejo desconocían, ocasionaron que la propuesta no se realizara por un par de años, hasta que se vinculó con una prueba piloto en el marco de un proyecto de la Organización de las Naciones Unidas para la Alimentación y la Agricultura (FAO, por sus siglas en inglés), entre 2005 y 2007, denominado Diversificación de la Agricultura en Guatemala y México (Chiapas) a través de la Producción y Exportación de Frutas (puesto en marcha al mismo tiempo en las regiones Boca Costa y Petén de Guatemala). El objetivo fue aliviar "la pobreza rural a través de un modelo de reconversión productiva combinando una cadena de abastecimiento de fruta tropical con sistemas intensivos de producción de hortalizas" (Chang, Brattlof y Ghukasyan, 2014, p. 10). ${ }^{5}$ Los autores del reporte del proyecto consideraron favorable la intervención de la FAO, porque se "implementó de manera exitosa la conversión de un sistema tradicional de cultivo de café y maíz basado en pequeños productores, hacia una horticultura de alto valor basada en frutas y hortalizas" (Chang, Brattlof y Ghukasyan, 2014, p. 10). El proyecto retomó al pie de la letra los principios de reestructuración agrícola para el sur global.

Como resultado de su operación, "el proyecto mejoró las condiciones de vida de 3500 PP que se organizaron en 75 cooperativas" (p. 10). Respecto a la mejora de las condiciones de vida de los pequeños productores de Chiapas, el reporte menciona que "el Gobierno del Estado de Chiapas posteriormente invirtió 60 millones de dólares para replicar los modelos exitosos de organización y producción bajo el proyecto" (Chang, Brattlof y Ghukasyan, 2014, p. 12). Uno

5 Entre las razones para sembrar limón persa, la encuesta indicó lo siguiente: $48 \%$ de los productores lo hizo por los mayores ingresos recibidos; $32 \%$ debido a los apoyos de gobierno o de la FAO; $11 \%$ porque requería menor esfuerzo o cuidados en comparación con el maíz; y 9\% porque se lo recomendó otro productor. 


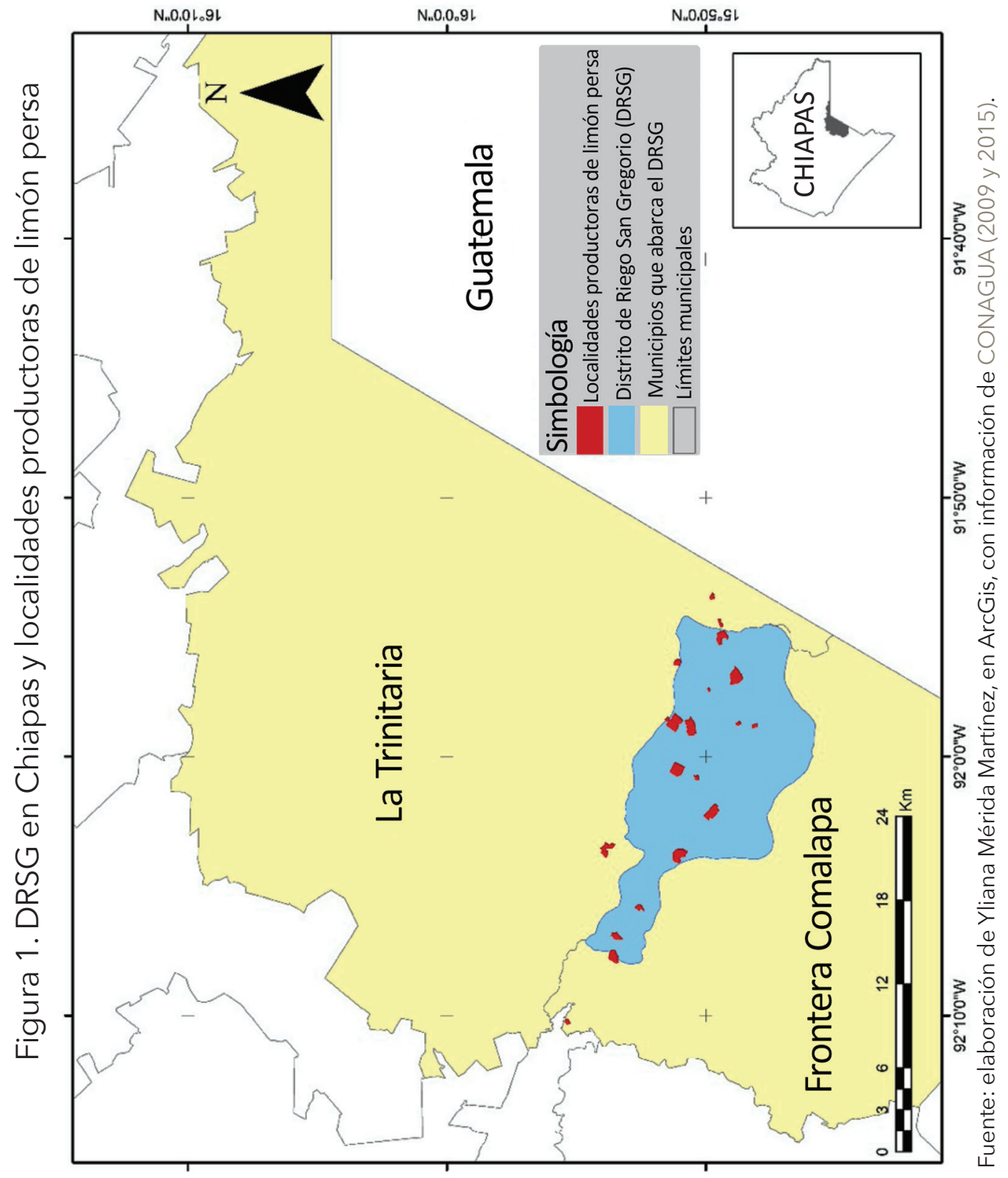


de los grupos formados se integró por 27 productores de las localidades Nuevo Villaflores y Flor de Mayo, a quienes se les proporcionaron árboles de limón persa, un invernadero para producir tomate saladette, equipo y asesoría técnica para producir ambos cultivos (notas de campo; Chang, Brattlof y Ghukasyan, 2014). Cuando al segundo año se obtuvieron cosechas, el producto se vendió en los mercados públicos de Comitán y Frontera Comalapa (P. Méndez, comunicación personal, 17 de mayo de 2016).

El Instituto para la Reconversión Productiva y la Agricultura Tropical (IRPAT), creado en 2008 durante la administración estatal de Juan Sabines Guerrero (Fletes et al., 2014), impulsó en el Distrito de Riego la conformación de cooperativas de citricultores mediante el ofrecimiento de recursos productivos, como insumos, equipo y asesorías. Entre las asociaciones que surgieron en el DRSG, se encuentran Cítricos Valle de Grijalva, Frutales Juan Sabines, Cooperativa de Citricultores San Isidro, Socios Limoneros de La Revolución y Sociedad de Citricultores Nuevo Amanecer. Este proceso de formación de organizaciones, como han documentado Jentoft y Finstad (2018, p. 14) y Selicato y Piscitelli (2016, p. 79), establece y representa los primeros esfuerzos de acción colectiva que permitiría integrar y coordinar las iniciativas de los actores locales desde el territorio una vez que se retiraran los apoyos.

Por su parte, la entonces Secretaría de Agricultura, Ganadería, Desarrollo Rural, Pesca y Alimentación (SAGARPA) y el Instituto de Reconversión Productiva y Bioenergéticos (IRBIO), agencia que se creó en 2010 con el objetivo de impulsar la reconversión productiva, integraron en 2011 el Comité Estatal del Sistema Producto Limón Persa en el estado de Chiapas y los comités regionales, con los cuales se planteó impulsar la producción del limón persa en el estado. A través del CRSPL se gestionaron ante diversas dependencias insumos, capacitaciones y una empacadora con la cual los productores lograron enviar tres embarques a Estados Unidos.

La inversión total reportada para la reconversión productiva entre 2007 y 2010 fue de 1160 millones de pesos para el estado y unos 157 millones de pesos en la región (Chang, Brattlof y Ghukasyan, 2014). La intervención de esos actores, los apoyos institucionales, las condiciones climáticas y la infraestructura de los canales de riego fueron las acciones y las condiciones que permitieron la introducción y la expansión del limón persa en el Distrito de Riego. Sin embargo, la instalación del cítrico, la implementación de las prácticas de cultivo y la definición de las estrategias comerciales correspondieron a los actores locales, lo que manifestó un "crecimiento de la sociedad local" que apuntaló procesos de desarrollo territorial (Selicato y Piscitelli, 2016, p. 79).

Prácticas de producción, conocimiento local

e innovación en torno al limón persa

Al constituir un proceso productivo con recursos, organización y conocimientos distintos a los que los productores habían incorporado con la producción de maíz, la introducción del limón persa en el Distrito de Riego requirió que emprendieran procesos de aprendizaje, formal y práctico. Como menciona Bueno 
(2012), las regiones con densidad de relaciones y capacidad para generar las bases de un "capital colaborativo" pueden enfrentar de mejor manera los retos externos y endógenos. El capital colaborativo no se construye sólo con la transmisión de conocimiento codificado, sino que también debe haber condiciones para compartir experiencias estimuladas por las relaciones informales (Bueno, 2012, p. 192). A través de este conocimiento tácito y de las relaciones informales, los productores desarrollaron las capacidades tecnológicas apropiadas para obtener productos que pudieran ser comercializados. Se evidenció cuando, al implementarse el CRSPL y retirarse las agencias y apoyos, los productores locales tomaron por su cuenta la trasmisión de conocimientos, tanto a los miembros de sus familias como a otros productores que deseaban ingresar en la actividad. Mediante estas estrategias lograron la innovación en la manera de hacer las cosas, adaptando las tecnologías externas a sus propias instituciones y condiciones locales. Como afirman Mazzoleni y Nelson (2011):

en virtualmente todos los campos de la práctica humana, el desempeño efectivo requiere más que la adquisición de capacidades tecnológicas en el sentido estrecho del término, lo que podríamos llamar tecnologías "físicas" [...]. Podría ser útil pensar el desempeño efectivo como dependiendo del dominio de tecnologías "sociales" relevantes, así como también en las tecnologías "físicas" involucradas. Las tecnologías sociales se acuerpan en formas organizacionales, cuerpos de ley, políticas públicas, códigos de buenas prácticas de negocios y administrativas, costumbres, normas. (p. 67)

En el proyecto de la FAO, y durante el fomento de la hortofruticultura por parte del IRBIO, un equipo técnico se encargó de impartir cursos y talleres a los productores sobre siembra, poda, fertilización, combate de plagas y cosecha de limón. Se instruyó también sobre aspectos concretos, como la distancia adecuada entre matas y surcos, de manera que se tuviera la densidad adecuada para el crecimiento de cada árbol, así como el tránsito en los pasillos para fumigar, fertilizar y cortar.

El manejo del cultivo conllevó que los productores modificaran la siembra, el control de plagas y la fertilización para mejorar la calidad y los rendimientos productivos. También se estableció la fumigación para controlar plagas, y la fertilización se realizaba en las primeras horas de la madrugada. Decidieron evitar la siembra de maíz en las huertas de limón durante los dos primeros años de crecimiento de los árboles, ya que, si bien el maíz permitía obtener alimento, reducía la luz solar que el árbol requería para su crecimiento. De esta forma, a partir de la práctica y del "aprender haciendo" en un contexto de incertidumbre (Mazzoleni y Nelson, 2011, p. 73), los productores no sólo afianzaron los conocimientos adquiridos en los talleres y capacitaciones, sino que también produjeron nuevos conocimientos que les permitieron mejorar sus sistemas de producción.

A partir de 2011, las dependencias de gobierno dejaron de impartir cursos y talleres de capacitación sobre el manejo del limón persa. Los productores tuvieron que buscar orientación de los citricultores con mayor experiencia para 
comenzar a producir y solucionar los problemas de producción. Aquéllos con mayor experiencia en el limón apoyaron a los que solicitaban asistencia para aprender a producir con base en las relaciones de amistad que sostenían y en el reconocimiento de que la incorporación de nuevos citricultores en la región no creaba competencia entre ellos ni saturaba el mercado, sino que, al contrario, los beneficiaba debido a que el mayor volumen disponible motivaba a los comercializadores foráneos a seguir acudiendo a la región.

En resumen, los resultados de la encuesta indican que los talleres con FAO e IRBIO constituyeron la principal forma de aprendizaje para $71 \%$ de los productores. Después se encuentra el diálogo con los productores de mayor experiencia (21\%). Pero cuando los apoyos y los talleres del gobierno estatal se redujeron, la trasmisión de conocimientos de productor a productor fue la principal forma de aprendizaje en $65 \%$ de los casos, lo que tiene un papel fundamental en vista de la falta de asistencia técnica contra plagas y enfermedades. ${ }^{6}$ También $23 \%$ de los productores acudió con los ingenieros de CESAVE, y $9 \%$ con los vendedores de insumos agrícolas.

La disposición para compartir conocimiento entre los productores es resultado de las relaciones de confianza y de los beneficios monetarios que perciben. Los productores actuaron de forma colectiva para el control de plagas, y el trabajo que cada productor realizaba en su parcela para cuidar sus cultivos (y el apoyo que daban a otros productores) evitaba la propagación de las plagas en las parcelas contiguas y en el Distrito en general. Así se evidencia la importancia de las relaciones de cooperación para el logro de objetivos comunes. Este tipo de economía externa -como se ha señalado en la literatura sobre los distritos industriales (Carreto, 2013, pp. 97-99) - resultó fundamental para alcanzar un cierto grado de coordinación e incorporar a nuevos citricultores en la región. Se ha encontrado que este proceso de sinergia y de coordinación como grupo o comunidad local mejora el desempeño de todos los participantes (Jentoft y Finstad, 2018, p. 14) no sólo en la producción, sino también en el comercio.

Es importante hacer una acotación respecto al impacto ambiental de las prácticas de cultivo asociadas con esta nueva plantación. En el trabajo de campo se observó el uso elevado de agroquímicos en la producción del cítrico. La totalidad de los citricultores utilizó fertilizantes químicos, entre los que destacan Triple 17 y urea. El herbicida glifosato fue utilizado por $60 \%$ y 10\% limpió con machete. De acuerdo con los ingenieros de CESAVE-Chiapas (julio, 2015), debido a la aplicación inadecuada de los fertilizantes granulados antes del riego, es frecuente que en las parcelas crezcan plantas con diversos tipos de clorosis por la deficiencia de micronutrientes y macronutrientes, lo cual ocasiona una reducción de la productividad y de calidad del producto. La Campaña Contra Plagas de los Cítricos del CESAVE afirma también que, debido a la pérdida en el equilibrio natural en el cultivo de cítricos, se ha observado la prolifera-

6 Esta forma de aprendizaje permitió solventar un problema señalado en un estudio sobre competitividad del limón persa en la región del Papaloapan, Oaxaca (López-Hernández, Garza-Bueno, Cruz-Galindo y Nieto-Ángel, 2019, p. 926), en donde se encuentra un porcentaje mucho más bajo de productores que recibieron capacitación técnica en el periodo que analiza (33\%, entre los años 2017 y 2018$)$, lo que de alguna manera afectaba sus niveles de productividad. 
ción de plagas secundarias, como la mosca prieta (Aleurocanthus woglumi). En el caso del Distrito de Riego, donde 70\% de los citricultores usó agroquímicos para controlar las plagas de mosca prieta y mosca blanca (notas de campo), el uso inmoderado de plaguicidas ha causado la reducción de la fauna benéfica, encargada de controlar la población de esta mosca, lo cual creó las condiciones para su multiplicación.

Considerando estos hallazgos, el uso extendido que los productores de limón persa hacen de los agroquímicos, representa una amenaza importante a su salud y a la de sus familias. Cabe señalar que esta práctica de los PP no es exclusiva de la producción de limón, sino más bien una característica de la producción del DRSG. Diversos productores comentan que cada vez tienen que utilizar una mayor cantidad de agroquímicos para mantener los rendimientos, porque las plagas son más resistentes y con el tiempo la tierra va perdiendo su productividad (notas de campo). Sobre esto, González (2020, p. 174) encontró que la producción de $\mathrm{FH}$ en México ha conllevado la utilización de métodos intensivos de producción que impactan de manera negativa en el medio ambiente y en la sociedad, porque provocan la erosión del suelo, la contaminación del aire, del agua y del suelo, el incremento de plagas y el surgimiento de problemas de salud en los productores, jornaleros y en la población en general donde se siembran estos cultivos.

Organización para la comercialización de limón persa

La coordinación de los productores para vincularse con intermediarios comerciales foráneos, así como sus estrategias para vender el cítrico en Guatemala, abrió canales de comercialización mediante los que se obtuvieron ingresos constantes y altos en términos relativos en ciertas temporadas del año. Cuando se presentaron las intervenciones de la OPEZ y de la FAO, los citricultores vendieron las primeras cosechas de limón persa en los mercados públicos de Comitán de Domínguez y de Frontera Comalapa, como es el caso del mercado Unión de Productores Independientes de la Región Fronteriza, S. P. R. de R. L., ubicado en el primer municipio antes mencionado (Fletes et al., 2016; Fletes y Ocampo, 2018). Pero en 2007, un trabajador de una empresa empacadora de Tabasco contactó a un productor de la localidad Nuevo Villaflores después de observar las parcelas desde la carretera internacional Trinitaria-Frontera Comalapa y comenzó a comprar limón persa a los productores locales durante la temporada de precios altos, es decir, entre noviembre y abril (P. Méndez, comunicación personal, 17 de mayo de 2016).

Así pues, el limón persa de los PP del Distrito se vende a través de dos principales canales de comercialización. El de primera calidad se dirige a las empacadoras de Veracruz y de Tabasco, que lo distribuyen en el mercado nacional e incluso exportan a Estados Unidos (sobre la cualidad exportadora de México con respecto al limón persa, véase tabla 2), y venden el de segunda calidad a Guatemala y al mercado regional. 
Tabla 2. México. Superficie, producción y exportación de limón persa, 1999-2019

\begin{tabular}{|c|r|r|r|c|c|c|c|c|}
\hline \multirow{2}{*}{ Año } & \multicolumn{3}{|c|}{$\begin{array}{c}\text { Superficie sembrada } \\
\text { (ha) }\end{array}$} & Producción & Rendimiento & $\begin{array}{c}\text { Precio } \\
\text { medio rural }\end{array}$ & $\begin{array}{c}\text { Volumen de } \\
\text { exportación }\end{array}$ & $\begin{array}{c}\text { Porcentaje de } \\
\text { exportación }\end{array}$ \\
\cline { 2 - 10 } & Riego & Temporal & Total & (ton) & (ton/ha) & (pesos) & (ton) & (\%) \\
\hline 1999 & 3631 & 20942 & 24573 & 242133 & 12.04 & 2207.88 & Nd & \\
\hline 2004 & 8700 & 38592 & 47293 & 642430 & 14.21 & 1603.35 & 312554 & 48.7 \\
\hline 2009 & 13207 & 48615 & 61822 & 823220 & 14.13 & 2082.36 & 395198 & 48.0 \\
\hline 2014 & 21465 & 62983 & 84448 & 1066442 & 14.30 & 3732.28 & 451919 & 42.4 \\
\hline 2019 & 3166 & 67846 & 99512 & 1325385 & 14.16 & 5686.19 & 678851 & 51.2 \\
\hline
\end{tabular}

Fuente: elaboración propia con información de los Fideicomisos Instituidos en Relación con la Agricultura (FIRA, 2020) y del Sistema de Información Arancelaria Vía Internet (SIAVI, 2020).

El limón de primera calidad o de exportación se caracteriza por tener un color verde oscuro, un tamaño pequeño, una textura rugosa y por estar libre de manchas de plagas, condiciones que alargan su vida en el anaquel. Álvarez (2012, p. 67) encontró situaciones similares en Martínez de la Torre (Veracruz), en donde la calidad de limón persa se basa en el tamaño y el color. El verde más oscuro corresponde al estándar de los mercados japonés y europeo. El fruto con un verde más ligero se lo denomina limón de exportación y se dirige a Estados Unidos. El tono más pálido o amarillento se dirige al mercado nacional. En ese sentido, el conjunto de relaciones con las empacadoras veracruzanas y tabasqueñas da cuenta de la forma que adquiere la integración global en ese territorio, en la medida en que los estándares de calidad que se manejan en las cadenas largas repercuten sobre las dinámicas agrícolas de los PP del DRSG.

La atracción de los compradores hacia el producto de la región se ha basado en ciertas ventajas naturales y en la capacidad organizativa de los productores. En México, la reducción de las lluvias entre noviembre y abril ocasiona una contracción de la oferta nacional y, en consecuencia, se aumentan los precios, lo que motiva a las empacadoras y a los distribuidores nacionales a buscar fuentes de abastecimiento en regiones como el DRSG, donde el riego permite su disponibilidad todo el año. En ocasiones, fuertes lluvias causadas por fenómenos naturales y la propagación de plagas han mermado la producción de las principales regiones limoneras del país, como Michoacán, Oaxaca, Colima y Veracruz (véase tabla 3 en referencia a los principales estados productores del país), lo que provoca un fuerte desabasto nacional y el incremento considerable de precios. Cuando estos fenómenos meteorológicos no han afectado el DRSG, los citricultores locales han obtenido altos precios, como ocurrió a finales de marzo e inicios de abril de 2014, cuando los productores de esta región vendieron a 35 pesos el kilogramo de limón de primera calidad a los comerciantes de Veracruz debido al desabasto. De acuerdo con Manuel y Pablo, productores de Nuevo Villaflores, que también fungen como comercializadores, ese precio por kilogramo 
era alto en comparación con lo que se había pagado en 2013 (10 pesos), o con el precio medio rural registrado en 2014 , de 8.1 pesos por kilogramo.

Tabla 3. México. Principales estados productores de limón persa, 2019

\begin{tabular}{|c|c|c|c|c|c|}
\hline \multirow{2}{*}{ Estado } & $\begin{array}{c}\text { Superficie } \\
\text { sembrada }\end{array}$ & $\begin{array}{c}\text { Superficie } \\
\text { cosechada }\end{array}$ & Producción & Rendimiento & $\begin{array}{c}\text { Precio medio } \\
\text { rural }\end{array}$ \\
\cline { 2 - 6 } & \multicolumn{2}{|c|}{ (ha) } & (ton) & (ton/ha) & (pesos/ton) \\
\hline Veracruz & 47831 & 46893 & 701904 & 15.0 & 5916.6 \\
\hline Oaxaca & 15056 & 14115 & 181699 & 12.9 & 5459.4 \\
\hline Jalisco & 5743 & 4714 & 88570 & 18.8 & 6827.1 \\
\hline Tabasco & 7227 & 7221 & 85941 & 11.9 & 3613.5 \\
\hline Yucatán & 3985 & 3369 & 70142 & 20.8 & 5234.1 \\
\hline Puebla & 2743 & 2562 & 32915 & 12.9 & 4554.1 \\
\hline Quintana Roo & 2376 & 2080 & 32505 & 15.6 & 5950.3 \\
\hline Michoacán & 3159 & 2796 & 31232 & 11.2 & 5846.1 \\
\hline Nayarit & 2713 & 2363 & 23291 & 9.9 & 4936.1 \\
\hline Campeche & 2099 & 1949 & 16918 & 8.7 & 4129.7 \\
\hline Chiapas & 2671 & 2363 & 13498 & 5.7 & 3647.2 \\
\hline
\end{tabular}

Fuente: FIRA (2020).

En la configuración de las relaciones comerciales fue también importante la acción colectiva. Para comercializar con los intermediarios foráneos, los PP tuvieron que organizarse para abastecer de manera colectiva, en tiempo y forma, el volumen requerido. Los comercializadores acuden cada quince días al Distrito con camiones acondicionados para cargar, en un máximo de dos días, 17 toneladas (unas 700 cajas de $25 \mathrm{~kg}$ ) de limón de primera calidad. En promedio, cada productor puede cosechar cada dos semanas 30 cajas de $25 \mathrm{~kg}$ de ese limón, por lo que se necesita el producto de aproximadamente 25 citricultores.

La necesidad de satisfacer en tiempo y forma el volumen que requieren los comercializadores foráneos motivó a ciertos productores a trabajar de intermediarios. Ellos se encargan de informar a los comercializadores la disponibilidad del limón en el Distrito y acuerdan el precio y la fecha en la que deberán acudir a la región para comprar el producto. A partir de esto, avisan a los productores el precio y la fecha pactada para que cosechen en el momento adecuado y cumplan con la cantidad deseada. De esta forma se evita que otros compradores foráneos acudan a la región en las mismas fechas y se queden sin la cantidad de producto que requieren.

Al mismo tiempo se constituyeron otros circuitos de comercialización de limón de primera calidad. En 2011, un comerciante ligado a una empresa de Ve- 
racruz instaló una bodega de acopio denominada Empacadora GONARCHI, Citricultores de Valle de Chamic, S. C. de R. L. de C. V., y en 2015 un comisionista de Tabasco instaló la empacadora Benavides, en la que acopia y empaca el limón de primera calidad de la región para enviarlo a la empresa Colimex, Inc., de Texas (Estados Unidos). ${ }^{7}$ Así mismo, desde 2014 un productor-comercializador de Nuevo Villaflores ha vendido en diversas ocasiones el producto de manera directa a empacadoras de Martínez de la Torre (Veracruz) a un precio mayor que el ofrecido en la región por los propios intermediarios foráneos ( $F$. Espinosa, comunicación personal, 30 de mayo de 2016). En 2015, el Gobierno del Estado entregó instalaciones para empaque a la Sociedad Cooperativa de Citricultores San Isidro. Ante la insuficiencia de equipo, en 2016 esos productores se asociaron con un fabricante de empacadoras y comercializador de frutas, a través del cual enviaron limón a Veracruz y exportaron a Estados Unidos (notas de campo).

Por su parte, los PP que se han convertido en comerciantes locales y los comerciantes foráneos instalados en la región venden el limón de segunda calidad (de color verde claro, de mayor tamaño, textura lisa y mayor perecibilidad) a intermediarios de Guatemala, y en menor medida a las centrales de abasto de Comitán de Domínguez y de Frontera Comalapa. La comercialización del limón persa hacia Guatemala comenzó en 2010 , cuando un par de pequeños productores originarios de Nuevo Villaflores acopiaron el producto de las localidades de la región y se contactaron con compradores de ese país. Eso ha motivado a otros PP a buscar sus propios canales de venta hacia el vecino país de Centroamérica y a convertirse en comercializadores regionales. Los dos comercializadores foráneos instalados en la región vieron en el mercado de Guatemala una forma de operar durante todo el año, porque la venta del limón de primera calidad hacia Veracruz y Tabasco sólo les era rentable entre noviembre y abril.

Una acción colectiva que apuntaló este proceso es el control que los productores ejercen sobre la comercialización hacia Guatemala, vigilando, en una caseta del poblado Chamic, que el limón persa vendido a ese país sea sólo el originario de las poblaciones del DRSG. Siguiendo el ejemplo del control del comercio de maíz que se realiza desde mediados de la década de 2000, los productores de limón persa tomaron esta decisión a partir de que un productor de la localidad de Nuevo Villaflores comercializó limón persa de Veracruz hacia Guatemala. Por esa razón, los actores locales protegen para sí el mercado centroamericano. ${ }^{8}$

Aunque el precio del kilogramo de limón de segunda calidad es menor que el de primera, los comerciantes regionales y los pequeños productores en general consideran que el mercado de Guatemala es el más importante, porque demanda limón durante todo el año y genera ingresos constantes para los citricultores

7 Colimex Inc. es una empresa de propiedad y gestión familiar de productores de limón persa originarios de Michoacán, ubicada en Texas (Estados Unidos). Aplicando las buenas prácticas agrícolas (GAP, por sus siglas en inglés), se dedica a la producción, compra y selección de limón persa, lima, tomatillo, papaya, coco y pimientos (http://www.colimexinc.com/).

8 La cualidad de flujos informales de limón hacia Guatemala se puede ver en que el sitio SIAVI de la Secretaría de Economía tiene vacíos de información sobre la exportación desde México hacia ese país. En 2016 y 2017 se reportaron 22.4 toneladas anuales de exportaciones y entre enero y abril de 2020, 58.3 (SIAVI, 2020). 
cada dos o tres semanas. Puesto que es considerado un activo que aporta un flujo monetario constante a las familias, un productor afirma:

una matita de limón es como una vaquita, porque cuando no hay dinero y hace falta algo en la casa, como el café o azúcar, va uno [a la parcela] y corta un poco de limón y lo vende para tener paga para comprar en la tienda. (R. Hernández, comunicación personal, 21 de mayo de 2016)

Las acciones colectivas llevaron a superar uno de los retos fundamentales que tienen los PP: el acceso a los mercados (Mutonyi, 2019). Tales acciones han derivado en el fortalecimiento de los conocimientos y de las capacidades de comercialización, mediante las cuales los productores locales se apropian de un mayor valor en la cadena productiva al reducir la dependencia de los intermediarios y comercializar por cuenta propia sus productos. ${ }^{9}$ Los mecanismos de coordinación que se han creado demuestran la cualidad social e institucional del mercado (Hodgson, 2011). ${ }^{10}$ De acuerdo con un comercializador local (y productor):

Cuando está bajo el precio, unos vendemos para Guatemala y otros mejor se van para Comitán. Así evitamos que todos vayamos por el mismo lado y que la gente, al ver mucho, nos quiera dar menos [...]. Porque si todos vamos a Comitán, rápido lo llenamos de limón y ya no vendemos. (F. Espinosa, comunicación personal, 30 de mayo de 2016)

\section{Conclusiones}

La acción colectiva con base en la comunidad y en las organizaciones de los pequeños productores conduce al logro de los objetivos comunes y es una herramienta para enfrentar los retos que presentan el desarrollo territorial y los cambios del sector alimentario regional y global. Con esa acción se supera el estancamiento relativo de la actividad agrícola respecto al resto del sistema económico.

Se encuentra que, si bien la introducción y la propagación de limón persa comportan intervenciones de actores diversos, fueron los productores locales quienes establecieron la acción colectiva desde el territorio. Es decir, en el

9 Predomina en los productores la opinión favorable con respecto a la relación comercial que tienen con el mercado de Guatemala y con la venta de limón de primera calidad a las empacadoras de Veracruz y de Tabasco en temporada de estiaje. De los encuestados, $88 \%$ señala estar satisfecho con los ingresos que genera esta actividad. Entre las razones por las cuales los productores perciben insatisfacción, se encuentran el tiempo de dos años que tarda la primera cosecha y la reducción de los precios de junio a octubre. La experiencia en el DRSG difiere de otros casos; por ejemplo, en el estado de Nayarit, en donde se encuentra que la "articulación de acciones" a través de las organizaciones "sigue siendo endeble, lo que perpetúa la condición de los productores y limita su papel en el desarrollo de sus comunidades" (Partida y Meza, 2017, p. 172).

10 "El mercado en sí es una institución, involucrando reglas complejas. En realidad, los mercados involucran normas sociales y costumbres, relaciones de cambio instituidas, y redes de información que tienen que explicarse" (Hodgson, 2011, p. 108). 
origen del proceso se puede identificar la influencia de un ente externo, lo cual es una posibilidad que han señalado Camacho (2008, p. 108) y Jentoft y Finstad (2018, p. 14). No obstante, los actores locales recuperaron elementos endógenos al territorio (confianza, valores y conocimientos comunes) para adoptar, adaptar y difundir varias técnicas de producción con el fin de realizar innovaciones sociotécnicas, cooperar y coordinarse para obtener cierto control de las redes comerciales que vincularon el producto con el mercado en diferentes escalas. Los mecanismos de coordinación de los propios productores dieron sustento a la acción. Esta situación difiere de otras experiencias, como las señaladas para los casos de la Cuenca del Papaloapan o de Nayarit. ${ }^{11}$ Pero se debe enfatizar que los elementos clave que la política pública podría alentar en otros espacios son precisamente la recuperación y la activación de los recursos endógenos al territorio, que no son exportables de un territorio a otro, pues obedecen a la historia cultural, productiva e institucional de un espacio concreto.

El estudio del caso registra que el programa de reconversión productiva representa una forma de concretar los principios de reestructuración agrícola para el sur global y que promueve la especialización en FH. Así, los productores se insertan en la división internacional del trabajo agrícola, característica del reciente proceso de globalización agroalimentaria. Debido a que se ha estimulado la orientación comercial intensiva de la agricultura del DRSG, a través de la aplicación generalizada de agroquímicos en la producción del cítrico, los productores y los actores institucionales locales detectan afectaciones en los ecosistemas y vulnerabilidad de la salud de la población.

El desempeño comercial de los PP se basó en el esfuerzo colectivo y en la definición de una oferta a nivel territorial. El proceso de sinergia y de coordinación del grupo y de la comunidad local mejora el desempeño de todos los participantes. De hecho, se demuestra la emergencia de un sistema productivo regional en torno al programa de reconversión. Las acciones emprendidas por los productores, en coordinación con los comercializadores foráneos para distribuir la producción de limón persa en los mercados nacional e internacional han permitido que la dinámica citrícola del Distrito de Riego supere la dificultad que por lo general representa la comercialización de productos agrícolas, sobre todo para los PP. En cierto sentido, el productor-comercializador local constituye un actor-agente, que es una "condición decisiva para el éxito de los procesos de desarrollo local” (Arocena y Marsiglia, 2017, p. 143). Esto constituye la base para movilizar el potencial de desarrollo y liderar el proceso de cambio territorial. Sin embargo, es importante que los grupos de actores locales integren otras dimensiones del desarrollo (social, cultural y ambiental) y sobre todo que eso se dé en un contexto en el que las agencias del Estado y otros actores del territorio se involucren de manera más consistente.

11 Véanse las notas 6 y 9. 


\section{Referencias}

Álvarez, R. (2012). Neoliberalism and the transnational activity of the State. Offshore control in the US-Mexico mango and persian lime industry. En T. Weaver. J. B. Greenberg, W. L. Alexander y A. Browning-Aiken (eds.), Neoliberalism and Commodity Production in Mexico (pp. 51-74). Boulder: University Press of Colorado. doi: https://doi.org/10.1111/amet.12124_22

Arocena, J., y Marsiglia, J. (2017). La escena territorial del desarrollo. Actores, relatos y políticas. Montevideo: Taurus y CLAEH.

Biel, R. (2007). Crisis y contradicciones en las relaciones Norte-Sur. México: Siglo XXI.

Biel, R. (2016). Sustainable Food Systems. The Role of the City. Londres: UCL. doi: $10.2307 /$ j.ctt1j1vzc5

Bonanno, A., y Wolf, S. (2018). Introduction. En A. Bonanno y S. Wolf (eds.), Resistance to the Neoliberal Agri-Food Regime. A Critical Analysis (pp. 1-18). Nueva York: Routledge y Earthscan Food and Agriculture.

Bueno, C. (2012). La selección de los más aptos: evolución de PYMES basadas en tecnología hacia la generación de soluciones productivas más complejas. En J. Carrillo, A. Hualde y D. Villavicencio (coords.), Dilemas de la innovación en México. Dinámicas sectoriales, territoriales e institucionales (pp. 185-224). México: El COLEF. Recuperado de https://colef.repositorioinstitucional.mx/ jspui/bitstream/1014/535/2/Dilemas\%20de\%20la\%20innovaci\%C3\%B3n--interiroes.pdf

Bueno, C. (2016). Configuraciones productivas en la globalización: trayectorias a la mexicana. México: CIESAS y UIA.

Camacho, J. (2008). Capital social y acción colectiva: estudio de casos de acción colectiva de productores lecheros de la cuenca de Tecamachalco, Puebla (tesis de maestría). Recuperado de https://www.colef.mx/posgrado/wp-content/ uploads/2010/11/TESIS-Camacho-Vera-Joaqu\%C3\%ADn-Huitzilihuitl-MDR.pdf

Carreto, J. (2013). Marshall y los sistemas productivos. Economía Informa (383), 90-106. doi: 10.1016/S0185-0849(13)71343-4

Castillo, V., Ayala, S., Jiménez, D., y Vargas, J. (2014). El comercio moderno: un vector que dinamiza el sistema alimentario en México. Revista de Economía del Caribe (13), 1-35. Recuperado de http: / /www.scielo.org.co/scielo.php?script=sci_abstract\&pid=S2011-21062014000100001

Chang, K., Brattlof, M., y Ghukasyan, S. (2014). Small holder participation in the tropical super fruits value chain: ensuring equitable share of the success to enhance their livelihood. Roma: FAO. Recuperado de http: / /www.fao.org / publications/card/en/c/85514b53-4645-5d56-9e9a-2a2d0cadf4ac/

Comisión Nacional del Agua (CONAGUA). (2009). Convenio de colaboración SGIH-OCFS-CHS-09-DR-192-RF-CC. DR 107 San Gregorio. Informe Final.

Comisión Nacional del Agua (CONAGUA). (2015). Programa Hídrico Regional 2014-2018 de la Región Hidrológica Administrativa XI Frontera Sur.

Eakin, H., Sweeney, S., Lerner, A., Appendini, K., Perales, H., Steigerwald, D. G., Dewes, C., Davenport, F., y Bausch, J. C. (2018). Agricultural change 
and resilience: agricultural policy, climate trends and market integration in the Mexican maize system. Anthropocene (23), 43-52. doi: /10.1016/j.ancene.2018.08.002

Fideicomisos Instituidos en Relación con la Agricultura (FIRA). (2020). Mercado Nacional. Limón persa. Recuperado de https://www.fira.gob.mx/InvYEvalEcon/EvaluacionIF

Fitting, E. (2014). Importar maíz, exportar mano de obra: régimen neoliberal del maíz, cultivos transgénicos y erosión de la biodiversidad en México. En G. Otero (coord.), La dieta neoliberal. Globalización y biotecnología agrícola en las Américas (pp. 151-179). México: Simon Fraser University, UAM-X y M. A. Porrúa.

Fletes, H., y Ocampo, G. (2018). Peasant resistance to the transnationalization of agriculture in Mexico's southern border. En A. Bonanno y S. Wolf (eds.), Resistance to the Neoliberal Agri-Food Regime. A Critical Analysis (pp. 135146). Nueva York: Routledge y Earthscan Food and Agriculture.

Fletes, H., Ocampo, G., y Valdiviezo, G. (2016). Reestructuración de la agricultura e inseguridad alimentaria. Las iniciativas y retos de los pequeños productores. Entre Diversidades. Revista de Ciencias Sociales y Humanidades, 1(7), 112-135. doi: 10.31644/ED.7.2016.a04

Fletes, H., Ocampo, G., y Valdiviezo, G. (2018). Amenazas de la globalización neoliberal a los territorios rurales y agrodiversidad. Procesos y alternativas. En G. Valdiviezo y G. Ocampo (coords.), Cambio socioterritorial y desarrollo local (pp. 15-32). México: UNACH y Colofón.

Fletes, H., Rangel, F., Oliva, A., y Ocampo, G. (2014). Revalorizar a los pequeños productores. Contribuciones y resistencias en una región agroexportadora de Chiapas. En H. Fletes, A. Macías y J. Madera (coords.), El papel de los pequeños productores en la agricultura y alimentación. La experiencia desde tres regiones agrícolas en México (pp. 93-151). México: Plaza y Valdés.

Flores, M. (2017). Seguridad alimentaria: un concepto multidimensional. En F. Torres (coord.), Implicaciones regionales de la seguridad alimentaria en la estructura del desarrollo económico de México (pp. 19-50). México: UNAM. doi: 10.22201/iiec.9786073006590e.2018

Francisco, R. A. (2010). Collective Action Theory and Empirical Evidence. Nueva York: Springer. doi: 10.1007/978-1-4419-1476-7

Garrapa, A. M. (2018). Supermarket revolution y agricultura californiana: ¿un modelo en expansión? Interdisciplina, 6(14), 155-176. doi: 10.22201/ceiich.24485705e.2018.14.63385

González, H. (2020). What socioenvironmental impacts did 35 years of export agriculture have in Mexico? (1980-2014): a transnational agri-food field analysis. Journal of Agrarian Change, 20(1), 163-187. doi: 10.1111/joac.12343

González de la Rocha, M., y Villagómez, P. (2008). ¿Encuesta o etnografía? Avances y tropiezos en el estudio del intercambio social. En F. Cortés, A. Escobar y M. González (eds.), Método científico y política social. A propósito de las evaluaciones cualitativas de programas sociales (pp. 297-373). México: El Colegio de México. 
Guzmán, E. (2018). De maíces a maíces. Agriculturas locales, disputas globales. México: Juan Pablos Editor y Universidad Autónoma del Estado de Morelos.

Hodgson, G. (2011). Evolutionary and institutional economics as the new mainstream? En J. Aboites y J. Corona (coords.), Economía de la innovación y desarrollo (pp. 104-119). México: UAM-X y Siglo XXI.

Jentoft, S., y Finstad, B. (2018). Building fisheries institutions through collective action in Norway. Maritime Studies (17), 13-25. doi: 10.1007/s40152-0180088-6

López-Hernández, W. A., Garza-Bueno, L. E., Cruz-Galindo, B., y Nieto-Ángel, R. (2019). Competitividad del limón persa en la región del Papaloapan, Oaxaca. Revista Mexicana de Ciencias Agrícolas, 10(4), 921-934. doi: 10.29312/ remexca.v10i4.408

Lugo-Morin, D. (2013). La acción colectiva rural en los sistemas productivos dinámicos. Revista de Estudios Sociales, 47(septiembre), 157-168. Recuperado de http://journals.openedition.org/revestudsoc/8111

Luiselli, C. (2017). Agricultura y alimentación en México. Evolución, desempeño y perspectivas. México: UNAM y Siglo XXI.

Macías, A. (2017). Tú produce, del resto yo me encargo. Integración transnacional hortofrutícola en el sur de Jalisco, México. En H. González y M. Calleja (eds.), Dinámica territorial agroalimentaria en tiempos de globalización (pp. 181-218). México: U. de G. y UNACH. Recuperado de http://www.profmex. org/ciclosytendencias/vinculos/oportuno.pdf

Maya, C. (2017). Globalización y competencia: Sinaloa y la diversificación de los mercados hortícolas de exportación. Mexico: Plaza y Valdés.

Mazzoleni, R., y Nelson, R. (2011). Public research institutions and economic catch-up. En J. Aboites y J. Corona (coords.), Economía de la innovación y desarrollo (pp. 67-93). México: UAM-X y Siglo XXI.

Melucci, A. (1991). La acción colectiva como construcción social. Estudios Sociológicos, 9(26), 357-364. Recuperado de https://estudiossociologicos.colmex. $\mathrm{mx} /$ index.php/es/article/view/911/911

Mutonyi, S. (2019). The effect of collective action on smallholder income and asset holdings in Kenya. World Development Perspectives (14), 1-7. doi: 10.1016/j.wdp.2019.02.010

Ochieng, J., Knerr B., Owuor, G., y Ouma, E. (2018). Strengthening collective action to improve marketing performance: evidence from farmer groups in Central Africa. The Journal of Agricultural Education and Extension, 24(2), 169-189. doi: 10.1080/1389224X.2018.1432493

Partida, M. E., y Meza, R. E. (2017). La competitividad y la productividad del limón persa en Nayarit (México). Cuadernos del Claeh, 36(105), 127-E. Recuperado de http://claeh.edu.uy/v2/images/Publicaciones/Cuadernos/105/105_ PartidaZamora.pdf

Puyana, A., y Romero, J. (2008). El sector agropecuario mexicano: un decenio con el Tratado de Libre Comercio de América del Norte. Efectos económicos y sociales. En A. Puyana y J. Romero (coords.), El sector agropecuario y el Tratado de Libre Comercio de América del Norte. Efectos económicos y sociales (pp. 31-66). México: El Colegio de México. 
Reig, N. (2004). América Latina y la mundialización agroalimentaria. En M. del C. del Valle (coord.), El desarrollo agrícola y rural del tercer mundo en el contexto de la mundialización (pp. 23-54). México: UNAM y Plaza y Valdés. Recuperado de http://www.economia.unam.mx/academia/inae/pdf/inae2/ c2.pdf

Robinson, W. (2015). América Latina y el capitalismo global. Una perspectiva crítica de la globalización. México: Siglo XXI.

Rubio, B. (2006). Voces de la desesperanza: La desestructuración alimentaria en México (1994-2004). Gaceta Laboral, 12(1), 69-89. Recuperado de http:// www. redalyc.org/articulo.oa?id=33612104

Sekine, K., y Bonanno, A. (2016). The Contradictions of Neoliberal Agri-Food. Corporations, Resistance and Disasters in Japan. Morgantown: West Virginia University Press.

Selicato, F., y Piscitelli, C. (2016). Territorial cultural systems: possible definitions. En F. Rotondo, F. Selicato, V. Marín y J. López (eds.), Cultural Territorial Systems. Ladscape and Cultural Heritage as a Key to Sustainable and Local Development in Eastern Europe (pp. 75-84). Nueva York: Springer Geography. doi: 10.1007/978-3-319-20753-7_8

Secretaría de Medio Ambiente y Recursos Naturales (SEMARNAT) y Comisión Nacional del Agua (CONAGUA). (2017). Estadísticas agrícolas de los Distritos de Riego. Año Agrícola 2015-2016. México. Recuperado de https://files.conagua.gob.mx/conagua/publicaciones/Publicaciones/EA_2015-2016.pdf

Secretaría de Medio Ambiente y Recursos Naturales y Comisión Nacional del Agua. (2018). Estadísticas agrícolas de los Distritos de Riego. Año Agrícola 2016-2017. México. Recuperado de https://files.conagua.gob.mx/conagua/ publicaciones/Publicaciones/EA_2016-2017.pdf

Sistema de Información Arancelaria Vía Internet (SIAVI). (2020). Recuperado de http://www.economia-snci.gob.mx/siavi4/fraccion.php

Tisenkopfs, T., Adamsone-Fiskovica, A., Kilis, E., Šūmane, S., Grivins, M., Pinto-Correia, T., Bjørkhaug, H. (2020). Territorial fitting of small farms in Europe. Global Food Security, 26(septiembre), 1-9. doi: 10.1016/j.gfs.2020.100425

Torres, F. (2017). La seguridad alimentaria en la estructura del desarrollo económico de México. En F. Torres (coord.), Implicaciones regionales de la seguridad alimentaria en la estructura del desarrollo económico de México (pp. 119-176). México: UNAM. doi: 10.22201/iiec.9786073006590e.2018

Trápaga, Y. (2017). Factores estructurales de la seguridad alimentaria. En F. Torres (coord.), Implicaciones regionales de la seguridad alimentaria en la estructura del desarrollo económico de México (pp. 51-84). México: UNAM. doi: 10.22201/iiec.9786073006590e.2018

Vázquez-Barquero, A. (2015). El desarrollo endógeno en los territorios frágiles. La relevancia de las instituciones. En M. del P. Pérez, H. Merritt y G. Isunza (coords.), Los desafíos del desarrollo local (pp. 187-212). México: IPN y Miguel Ángel Porrúa.

Yúnez, A. (2018). La agricultura y el sector rural. En R. Campos y F. Hernández (coords.), Buen diagnóstico, buena solución. Los retos de la economía mexicana (pp. 103-115). México: FCE. 